\title{
Omadacycline efficacy against Streptococcus agalactiae isolated in China: in vitro activity, resistance analysis, clonality of MIC distribution, and the correlation between resistance and virulence gene and biofilm formation
}

\section{Guiqiu Li}

Shenzhen Nanshan People's Hospital

\section{Ying Wei}

The 6th Affiliated hosptial of Shenzhen university Healthy science center

Yan Guo

The 6th affilited hosptial of shenzhen uninversity health science center

Jie Lian

Shenzhen Nanshan people's hospital

\section{Shaoyuan Wu}

Shenzhen Nanshan people hospital

\section{Zhijian Yu}

Shenzhen Nanshan people's hospital

\section{Bing Bai}

Shenzhen Nanshan People's hospital

Qiwei Deng ( $\sim$ dqw139343@sina.com)

Shenzhen Nanshan People's Hospital. No 89, Taoyuan Road, Nanshan District, Shenzhen 518052, China

\section{Guangjian Xu}

Shenzhen Nanshan people's hospital

\section{Research}

Keywords: omadacycline, S. agalactiae, multilocus sequence typing, resistance genes, virulence genes

Posted Date: June 23rd, 2020

DOI: https://doi.org/10.21203/rs.3.rs-35559/v1

License: (c) (i) This work is licensed under a Creative Commons Attribution 4.0 International License. Read Full License 


\section{Abstract}

Purpose: This study aimed to evaluate activity『resistance, clonality of MIC distribution, and the correlation between virulence $₫$ resistance gene and biofilm formation of omadacycline (OMC) in clinical for Streptococcus agalactiae isolates from China.

Methods: 162 isolates were collected retrospectively in China from 2018 to 2019. The S. agalactiae were mainly collected from the body's cervical secretions, wound secretions, ear swab, secretions, semen, venous blood, cerebrospinal fluid, pee, urethral discharge, pus, umbilical secretions, wound secretions, reproductive tract secretions, sputum, gastric juice, throat swab, eye secretions and amniotic fluid. The MIC of OMC against $S$. agalactiae were determined by broth microdilution. Filter paper was used to measure the inhibition zone diameters of OMC and other common antibiotics. D-test detected the incidence of erythromycin resistance to inductively clindamycin. Multilocus sequence typing (MLST)\resistance $\$ virulence gene of the isolates were investigated using qRT-PCR. Biofilms were detected by crystal violet staining.

Results: The OMC MIC of clinical S. agalactiae isolates ranged from $\leq 0.25$ to $0.5 \mathrm{mg} / \mathrm{L}$. $19.7 \%$ of the $S$. agalactiae isolates with an OMC MIC of $0.25 \mathrm{mg} / \mathrm{L}$ were found to be sequence type (ST) 17. The S. agalactiae had highly resistant to Minocyclin, Erytromycin, Solithromycin and Clindamycin and the resistance rate of OMA was $13.6 \%$. The positive rate of D-test was $90.74 \%$. The formation of biofilm was related to scpB gene, and indicated the resistance of OMA may be related to the virulence gene scpB.

Conclusion: OMC exhibited better activity against clinical $S$. agalactiae isolates from China than DOX or MIN, and scpB was related with biofilm formation in OMC- resistance $S$. agalactiae.

\section{Introduction}

S. agalactiae belong to Group B Streptococcus (GBS) ${ }^{[1]}$. S. agalactiae is a kind of gram-positive cocci that microscopically examined for long-chain or long-chain chain arrangement. There are 10 serotypes in $\nabla, \nabla, \nabla, \nabla, \nabla, \nabla$, and $\mathbb{\triangle}^{[2]}$. S. agalactiae is ubiquitous in the natural environment and can reside in the human reproductive tract and digestive tract. It is one of the main pathogenic bacteria in humans and animals ${ }^{[3]}$. $S$. agalactiae is the most important pathogenic bacteria causing mastitis in dairy cows. S. agalactiae which has a strong infectivity, has also been isolated in the main culture area of tilapia. Human-derived $S$. agalactiae can cause morbidity or even death in newborns, pregnant women, the elderly, and immunocompromised persons. Human-derived S. agalactiae mainly causes endometritis in women and meningitis, sepsis and pneumonia in infants and young children, among which the infection rate of pregnant women is higher. Studies have reported that about $15-40 \%$ of adult women carry or are infected with S. agalactiae, and $25 \%$ passing on to babies of which $2 \%$ of infants show clinical symptoms, and the mortality rate can be up to $50 \%$, most of the surviving children have permanent neurological sequelae. The neonatal invasive infections caused by them have caused widespread concern worldwide.

In recent years, bacterial resistance and multi-drug resistance caused by a large number of clinically unreasonable use of antibiotics are becoming more and more common ${ }^{[4]}$, and the resistance and resistance spectrum of $S$. agalactiae are also increasing. Human-derived $S$. agalactiae is highly resistant to erythromycin, while tilapia is sensitive. Mataini et al ${ }^{[5]}$ found that $S$. agalactiae in Italy has the highest resistance rate to clindamycin, reaching $43.75 \%$, followed by erythromycin resistance rate of $32.20 \%$. 
S. agalactiae showed resistance to antibiotics mediated by corresponding resistance genes. The efflux pump mechanism, the ribosomal 23SrRNA methylation mechanism ${ }^{[6]}$, and the ribosomal protective protein effect mechanism ${ }^{[7]}$ regulated by the coding of the tet gene are the three main resistance mechanisms of $S$. agalactiae. The efflux pump mechanism is the main form of $S$. agalactiae anti-macrolide drugs, which is expressed in the form of a proton efflux pump mediated by mefA gene, which will expel the macrolide antibiotics out of the cell body, so that the target site drugs. The concentration is lower than the effective bacteriostatic concentration, thus showing resistance to 14- and 15-membered ring macrolide antibiotics. The ribosomal 23SrRNA methylation mechanism leads to resistance to erythromycin. The Erm gene carrying a rate of $72.7 \%$ encodes ribosomal methylase to methylate the ribosomal 23SrRNA at the target of erythromycin ${ }^{[8]}$. In turn, it is resistant to erythromycin.

Biofilm formation is one of the important reasons for bacterial resistance. In recent years, studies have shown that resistant $S$. agalactiae has become a relatively high proportion, but the relationship between resistant $S$. agalactiae gene and biofilm formation is rarely reported ${ }^{[9]}$. The distribution of these resistant and virulence factors in OMC-resistant $S$. agalactiae is unclear.

$\mathrm{OMC}$ is a new aminomethylcycline antibiotic first-in-class against Gram-positive and negative aerobic, atypical pathogens and anaerobic ${ }^{[10]}$. OMC has shown excellent efficacy in the treatment of acute skin, soft tissue infections ${ }^{[11]}$ and community-acquired pneumonia ${ }^{[12]}$. However,the resistance of OMC against isolates of $S$. agalactiae in clinical from China has not been reported ${ }^{[13]}$.

The purpose of the study was evaluating activity,resistance, clonality of MIC distribution, and the correlation between resistance $\llbracket$ virulence gene and biofilm formation of OMC-resistance $S$. agalactiae isolates in clinical from China.

We examined the MIC of OMC against S.agalactiae by broth microdilution. Filter paper was used to measure the inhibition zone diameters of OMC. The incidence of erythromycin resistance to inductively clindamycin were detected by D-test. We also used qRT-PCR to investigate MLST,virulencelresistance gene of the isolates. Biofilms were detected by crystal violet staining. The correlation between resistance $\square$ resistance genes and biofilm formation was calculated and observed.

\section{Materials And Methods}

\section{Bacterial isolates[culture and chemicals.}

163 non-duplicate clinical S.agalactiae strains were collected from patients at Shenzhen Nanshan People's Hospital from January 1, 2018 to December 31, 2019. Bacterial species were identified by standard methods using a VITEK 2 compact system (Biomérieux, Marcy l'Etoile, France). The S.agalactiae strains were cultured in $5 \%$ calf serum in TSB broth medium in $37^{\circ} \mathrm{C}$ constant temperature shaker for $18-24 \mathrm{~h}$ and then removed. The OMC

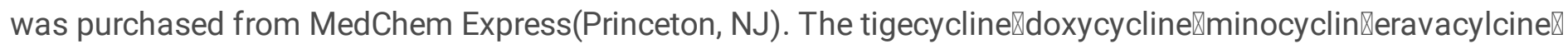

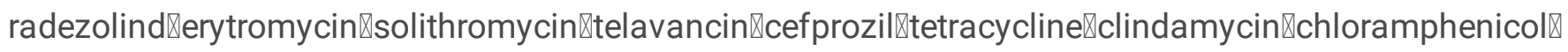
levofloxacin were purchased from Aladdin (Shanghai, China).

\section{Antibiotic susceptibility testing}


Antimicrobial susceptibility of $\mathrm{OMC}$ and several common antibiotics, including the tigecycline $\Downarrow$ doxycycline $\rrbracket$

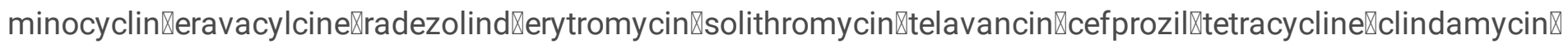

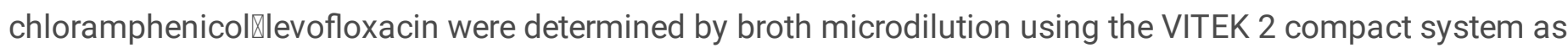
before. The MICs and Inhibition Zone Diameter of antibiotics were determined respectively according to the 2020 Clinical and Laboratory Standards Institute (CLSI) guidelines. The MIC breakpoint of omadacycline邓tigecycline

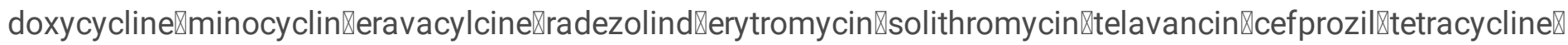
clindamycin $₫$ chloramphenicol『and levofloxacin were selected from 2020 CLSI. The MIC breakpoint of OMC:

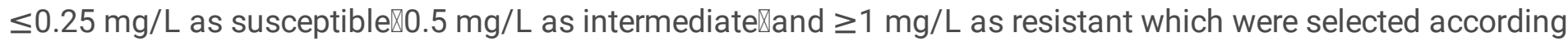
to previous literature.

\section{D-test for detectig the incidence of erythromycin resistance to inductively clindamycin}

Spread the S.agalactiae with a concentration of 0.5 McLaren's turbidity tube on the MH agar plate, applied erythromycin and clindamycin paper at $15 \mathrm{~mm}$ intervals, and placed it in a $35^{\circ} \mathrm{Cconstant}$ temperature incubator for 16-18 hours. When the bacteriostatic ring of clindamycin paper seemed to be a capital "D", it was judged as "D" test positive indicating that erythromycin had induced resistance to clindamycin.

\section{qRT-PCR analysis}

QRT-PCR was used for the analysis of MLST and the detection of resistance and virulence genes. Total bacterial RNA was extracted from S.agalactiae isolates, and reverse transcribed into cDNA which performed as previous literature we published. The primers of resistance and virulence genes were synthesized by Beijing Liuhe Huada Gene Company. Overnight cultures of the bacterial strains growing for $4 \mathrm{~h}$ at $37^{\circ} \mathrm{C}$. Extract the genomic DNA of the strain according to the instructions of the kit and store at $-20^{\circ} \mathrm{C}$ for future use.

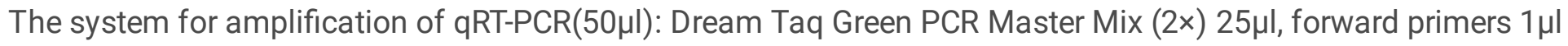
and reverse primers $1 \mu \mathrm{l}$, DNA $2 \mu \mathrm{l}$, added $\mathrm{ddH}_{2} \mathrm{O}$ to make up to $50 \mu \mathrm{l}$.

qRT-PCR reaction conditions: pre-denaturation at $95^{\circ} \mathrm{C}$ for $3 \mathrm{~min}$; denaturation at $95^{\circ} \mathrm{Cfor} 30 \mathrm{~s}$, annealing at $52^{\circ} \mathrm{Cfor} 30 \mathrm{~s}$, extension at $72^{\circ} \mathrm{Cfor} 1 \mathrm{~min}, 30$ cycles; $72^{\circ} \mathrm{Cfor} 10 \mathrm{~min}$.

The RNA were submitted to qRT-PCR after adding the qRT-PCR Master Mix (Thermo Fisher Scientific Waltham, MA, USA). The products of qRT-PCR were stored at $4^{\circ} \mathrm{C}$. The recA was used as internal control gene. The threshold cycle (Ct) numbers were analyzed using the $2^{-\triangle \Delta} \mathrm{Ct}$ method. The qRT-PCR reaction products were electrophoresed on 1\% agarose gel, and judged and analyzed according to the presence or absence of positive amplification products and the length of the target gene fragment. All qRT-PCRs were conducted in triplicate.

\section{Crystal violet staining for investigating resistancelvirulence gene of the isolates detection of Biofilms}

After bacteria grown overnight in TSB medium, diluted 200 and TSBG medium with $0.5 \%$ glucose. 200ul per well was added to 96 -well polystyrene microplates with 3 replicate wells, $37^{\circ} \mathrm{C}$ incubated at room temperature for 24 hours at static temperature. And then blotted the pores and rinsed with PBS 3 times, fixed with methanol for 15 minutes, stain with $0.5 \%$ crystal violet for 10 minutes, and rinsed with distilled water. Added $4: 1$ confluence solution of absolute ethanol and acetone, mixed evenly and tested under OD570 photometry in the end. OG1RF and CHS787 were used as the quality control strain. The interpretation of the OD value of the biofilm varies from 
0.05 to 3.5 in 96 microwells after staining. Biofilm phenotype classification was based on the method of others, strong positive (OD570> 2), moderate (OD 570, 1-2), or weak $(0.5<0 D 570<1)$.

\section{Statistical analysis}

The data was analysed using $t$-test by SPSS software. Significantly $P$ values $<0.05$.

\section{Results}

\section{Antimicrobial activity and resistance analysis of OMC against S.agalactiae isolates clinical in vitro}

The clinical S.agalactiae strains were isolated from various infective sample sources, including cervical secretions, wound secretions, ear swab, secretions, semen, venous blood, cerebrospinal fluid, pee, urethral discharge, pus, umbilical secretions, wound secretions, reproductive tract secretions, sputum, gastric juice, throat swab, eye secretions and amniotic fluid (Figue 1). The MIC and resistance rate of those isolates against antibiotic were obtained0Table 10, and OMC had in vitro resistance against S.agalactiae. OMC had robust antimicrobial activity against S.agalactiae in vitro, the clinical S.agalactiae isolates exhibited high-proportion resistance rate to Minocyclin, Erytromycin, Solithromycin and Clindamycin. The range of OMC MIC values against S.agalactiae was $0.25-1.0 \mathrm{mg} / \mathrm{L}$. The incidence of erythromycin resistance to inductively clindamycin against S.agalactiae was $90.74 \%$ (Table 2) \indicated that the incidence of inducing clindamycin resistance was high. The distribution of TET-specific resistance genes in clinical S.agalactiae isolates is shown in Table 3, the data suggested that the presence of tet(M) $\square \operatorname{tet}(0) \square \operatorname{tet}(\mathrm{K}) \square \operatorname{tet}(\mathrm{M})$, and tet(O) genes did not affect OMC sensitivity in S.agalactiae.

\section{Clonality of OMC MIC distribution}

Twenty-one STs were identified among the S.agalactiae isolated. The predominant STs were ST10 (32/162; $19.7 \%$ ) and ST17 (20/162; $12.3 \%$ ). A new typing had been detected. And $18.8 \%$ of ST10 strains and $25.0 \%$ of ST17 strains had an OMC MIC level of $1 \mathrm{mg} / \mathrm{L}$, demonstrated clonal clustering toward the ST17 genotype(Table 4 ).

\section{Formation characteristics of OMC-resistant S.agalactiae}

The 96-well plate readings after crystal violet staining ranged from 0.13 to 3.10. The median values of OG1RF strain and CHS787 control strain OD570 were 1.09 and 1.42, respectively. Among all the S.agalactiae isolates tested, most of the S.agalactiae biofilms showed weak positive and above. Among the strains formed by these biofilms, the weak biofilm phenotypes were different from the medium biofilm phenotypes and strong. The number of strains in the biofilm phenotype was similar.

\section{The correlation between virulencelresistance gene and biofilm formation}

PCR test results suggested that the OMC-resistant S.agalactiae resistance-positive genes ermB, ermC, OptrA, msrB, mefAE, tetM, tetO, tetK were detected, no ermA, cfr, cfrB, msrA gene-positive strains were detected(Table 5 ). The relationship between drug genes and biofilm formation still needed further study. There was no significant correlation between genes and biofilm formation by statistical analysis ( $P>0.05)$. 136 strains of S.agalactiae resistant to OMC, analysis of PCR amplified virulence genes showed that bac, bca, fbsA, fbsB, cfb, hylB, Imb, cylE, 
cpsA, rib, cpsIII, PI-1, PI-2a and PI-2b positive S.agalactiae strains were not statistically correlated with biofilm formation ( $P>0.05)$, while the scpB gene was just the opposite of its results, and it was correlated with biofilm formation by statistical analysis $(P<0.05)$ (Table 6$)$.

\section{Discussion}

In this study, OMC exhibited robust activity, resistance in vitro against S.agalactiae, and the clinical S.agalactiae isolates exhibited high resistance rate against Minocyclin, Erytromycin, Solithromycin and Clindamycin. The main STs observed were ST10 and ST17. The data indicated the clonality of S.agalactiae with clustering of the $1 \mathrm{mg} / \mathrm{L}$ OMC MIC in the ST17 genotype. The incidence of erythromycin resistance to inductively clindamycin against $S$. agalactiae was higher. So the clinical microbiology laboratory must strengthen the detection of the S.agalactiae D- test which is resistant to erythromycin and sensitive to clindamycin in order to guide the clinical rational use.

The OMC resistance genes ermA, rmB ${ }^{[14]}$, ermC, OptrA, msrB, mefAE, cfr, cfrB, msrA, tetM, tetO and tetK had no significant correlation with biofilm formation. Analysis of PCR amplified virulence genes showed that bac, bca, fbsA, fbsB, cfb, hylB, Imb, cylE, cpsA, rib, cpsIII, PI-1, PI-2a and PI-2b ${ }^{[15]}$ positive S.agalactiae strains have no significant correlation with biofilm formation by statistical analysis $(P>0.05)$, while the scpB gene was just opposite to its result, and it was correlated with biofilm formation by statistical analysis $(P<0.05)$.

In conclusion, OMC exhibited robust activity and resistance in vitro against S.agalactiae isolates. And OMC MIC with $1 \mathrm{mg} / \mathrm{L}$ showed ST17 clonality clustering. The incidence of erythromycin resistance to inductively clindamycin against $S$. agalactiae was high. The detection of the D-test for S.agalactiae must be strengthen in clinical because of its resistance. Biofilm formation ability and $\mathrm{OMC}$ resistance genes ermB, ermC, OptrA, msrB, mefAE, tetM, tetO, tetK, ermA, cfr, cfrB, msrA and virulence genes bca, bac, fbsA, fbsB, cfb, hylB, Imb, cylE, cpsA, rib, cpsIII, PI-1, PI-2a, PI-2b there was no significant correlation. ScpB was significantly associated with biofilm formation of S.agalactiae and OMC-resistant.

\section{Tables}

Table 1. Comparison of in vitro antimicrobial activity of OMC and various common antibiotics against $S$. agalactiae. 
Antibiotic

$\mathrm{N}$ of isolates(MIC /Inhibition

Zone Diameter)

\section{Total}

Tigecycline

$162 / n$

162

Doxycycline

Minocyclin

Eravacylcine

Omadacycline

Radezolind

Erytromycin

Solithromycin

Telavancin

162

$136 / 25$

Solithromycin 14

142
MIC(mg/l)

Resistance rate (\%)

MIC
(ug/ml) breakpoint
N Inhibition

Zone

Diameter(mm) ${ }^{a}$ breakpoint

\begin{tabular}{|c|c|c|c|c|c|c|}
\hline \multirow[t]{3}{*}{ Doxycycline } & \multirow[t]{3}{*}{162} & \multirow[t]{3}{*}{$0.25-22$} & \multirow[t]{3}{*}{22.2} & $\leq 0.25$ & 12 & - \\
\hline & & & & 0.5 & 4 & - \\
\hline & & & & $\geq 1$ & 110 & - \\
\hline \multirow[t]{3}{*}{ Minocyclin } & \multirow[t]{3}{*}{162} & \multirow[t]{3}{*}{$0.125-32$} & \multirow[t]{3}{*}{82.1} & $\leq 4$ & 23 & - \\
\hline & & & & 8 & 4 & - \\
\hline & & & & $\geq 16$ & 133 & - \\
\hline \multirow[t]{3}{*}{ Eravacylcine } & \multirow[t]{3}{*}{162} & \multirow{3}{*}{$\begin{array}{l}0.015- \\
0.25\end{array}$} & \multirow[t]{3}{*}{0} & $\leq 0.25$ & 162 & - \\
\hline & & & & 0.5 & 0 & - \\
\hline & & & & $\geq 1$ & 0 & - \\
\hline
\end{tabular}

$\begin{array}{lll}\leq 2 & 162 & - \\ 4 & 0 & - \\ \geq 8 & 0 & -\end{array}$

$\geq 8$

$0.25-0.5$

13.6

$\leq 0.25$

68

0.5

72

$\geq 1$

22

$\leq 2$

$162-$

4

$\geq 8$

$\leq 0.06-16$

$46.3 / 88.0$

$\leq 0.25$
0.5

$66 \geq 23$

1

$\geq 1$

$7 \quad 14-22$

2

$0.015-2 \quad 54.2$

$\leq 0.25$

$63 \leq 13$

22

\begin{tabular}{llll} 
& & \\
\hline Telavancin & 139 & $0.06-2$ & -
\end{tabular}

$\begin{array}{ll}0.5 & 38 \quad- \\ \geq 1 & 77\end{array}$

$\leq 0.12$

$24 \quad-$




\begin{tabular}{|c|c|c|c|c|c|c|c|}
\hline \multirow[t]{3}{*}{ Cefprozil } & \multirow[t]{3}{*}{142} & \multirow[t]{3}{*}{$0.004-2$} & \multirow[t]{3}{*}{0} & $\leq 2$ & 43 & - & - \\
\hline & & & & 4 & 0 & - & - \\
\hline & & & & $\geq 8$ & 0 & - & - \\
\hline \multirow[t]{3}{*}{ Tetracycline } & \multirow[t]{3}{*}{162} & \multirow[t]{3}{*}{-} & \multirow[t]{3}{*}{-} & $\leq 1$ & & - & - \\
\hline & & & & 2 & & - & - \\
\hline & & & & $\geq 4$ & & - & - \\
\hline \multirow[t]{3}{*}{ Clindamycin } & \multirow[t]{3}{*}{16} & \multirow[t]{3}{*}{-} & \multirow[t]{3}{*}{81.2} & - & - & $\geq 19$ & 2 \\
\hline & & & & - & - & $16-18$ & 1 \\
\hline & & & & - & - & $\leq 15$ & 13 \\
\hline \multirow[t]{3}{*}{ Chloramphenicol } & \multirow[t]{3}{*}{39} & \multirow[t]{3}{*}{-} & \multirow[t]{3}{*}{15.4} & - & - & $\geq 21$ & 11 \\
\hline & & & & - & - & $18-20$ & 22 \\
\hline & & & & - & - & $\leq 17$ & 6 \\
\hline \multirow[t]{3}{*}{ Levofloxacin } & \multirow[t]{3}{*}{16} & \multirow[t]{3}{*}{-} & \multirow[t]{3}{*}{25.0} & - & - & $\geq 17$ & 8 \\
\hline & & & & - & - & $14-16$ & 4 \\
\hline & & & & - & - & $\leq 13$ & 4 \\
\hline
\end{tabular}

Table 2. Drug resistnce rate of D-test against $S$. agalactiae.

\begin{tabular}{|c|c|c|c|c|}
\hline \multirow[t]{2}{*}{ Antibiotics } & \multirow[t]{2}{*}{$N$} & \multicolumn{2}{|c|}{ D-test } & \multirow[t]{2}{*}{ Drug resistnce rate } \\
\hline & & $D \square+\square$ & $D Q-D$ & \\
\hline Omadacycline & 162 & 15 & 147 & $90.74 \%$ \\
\hline
\end{tabular}

Table 3. The relationship among OMC, DOX, and MIN MIC distributions with ST10 and ST17 genotypes in S. agalactiae.

\begin{tabular}{|c|c|c|c|c|}
\hline \multirow{3}{*}{$\begin{array}{l}\text { Sequence } \\
\text { type }\end{array}$} & \multirow{3}{*}{$\begin{array}{l}N(\%) \text { of } \\
\text { isolates }\end{array}$} & \multicolumn{3}{|c|}{$N(\%)$ of isolates at each MIC level $(\mathrm{mg} / \mathrm{L})$} \\
\hline & & $\begin{array}{l}\text { ERAVMIC } \\
\text { distribution }\end{array}$ & $\begin{array}{l}\text { OMC MIC } \\
\text { distribution }\end{array}$ & $\begin{array}{l}\text { RADEMIC } \\
\text { distribution }\end{array}$ \\
\hline & & $\leq 0.25 \quad 0.5 \geq 1$ & $\leq 0.25 \quad 0.5 \geq 1$ & $\leq 2 \quad 4 \geq 8$ \\
\hline ST10 & $32(19.7)$ & $\begin{array}{llll}32(100) & 0(0) & 0\end{array}$ & $\begin{array}{l}9(28.1) \\
6(18.8)\end{array}$ & $32(100) 0(0) 0(0)$ \\
\hline ST17 & $20(12.3)$ & $\begin{array}{l}20(100) O(0) \\
O(0)\end{array}$ & $\begin{array}{l}9(45.0) \\
5(25.0)\end{array}$ & $20(100) 0(0) \quad 0(0)$ \\
\hline
\end{tabular}


Table 4. In vitro antimicrobial activity of OMC against $S$. agalactiae with TET-specific resistance genes

\begin{tabular}{|c|c|c|c|c|c|}
\hline \multirow[t]{2}{*}{$\begin{array}{l}\text { TET resistance } \\
\text { gene(s) }\end{array}$} & \multirow[t]{2}{*}{ No. } & & $\begin{array}{l}E R A V \\
\operatorname{MIC}(\mathrm{mg} / \mathrm{L})\end{array}$ & $\begin{array}{l}O M C \\
M I C(\mathrm{mg} / \mathrm{L})\end{array}$ & $\begin{array}{l}\text { RADE } \\
\operatorname{MIC}(\mathrm{mg} / \mathrm{L})\end{array}$ \\
\hline & & & $\begin{array}{l}\leq 0.250 .5 \\
\geq 1 \text { Range }\end{array}$ & $\begin{array}{l}\leq 0.250 .5 \\
\geq 1 \text { Range }\end{array}$ & $\begin{array}{l}\leq 24 \geq 8 \\
\text { Range }\end{array}$ \\
\hline $\operatorname{tet}(M)$ & 71 & 71 & $\begin{array}{lll}0 & 0 & 0.008-0.25\end{array}$ & $\begin{array}{l}32 \quad 2880.25- \\
1.00\end{array}$ & $-\quad-$ \\
\hline $\operatorname{tet}(0)$ & 46 & 46 & $\begin{array}{lll}0 & 0 & 0.008-0.25\end{array}$ & & $-\quad-$ \\
\hline $\operatorname{tet}(K)$ & 44 & 44 & $\begin{array}{lll}0 & 0 & 0.008-0.25\end{array}$ & $1.00^{21} \quad 0 \quad 0.20^{-}$ & $-\quad-$ \\
\hline $\operatorname{tet}(M), \operatorname{tet}(0)$ & 7 & 7 & $\begin{array}{lll}0 & 0 & 0.008-0.25\end{array}$ & $\begin{array}{l}1426 \quad 6 \quad 0.125- \\
1.00\end{array}$ & $-\quad-\quad-$ \\
\hline OptrA & 13 & - & -- & $\begin{array}{llll}4 & 0 & 2 & 0.25-\end{array}$ & $\begin{array}{l}1300 \\
0.25\end{array}$ \\
\hline None detected & $9 / 149$ & 9 & $\begin{array}{lll}0 & 0 & 0.015-0.25\end{array}$ & $\begin{array}{l}1.00 \\
-\quad-\quad-\end{array}$ & $\begin{array}{l}14900 \\
0.03-0.25\end{array}$ \\
\hline
\end{tabular}

Table 5. Relationships of OMC virulence genes with biofilm formation in S. agalactiae. 
OMC resistant Number and percentage of Streptococcus agalactiae $(\mathrm{N}=136)$

genes

N Strong Medium Weak Negative All positive

$\begin{array}{lllllllllll}\mathbf{N} & \% & \boldsymbol{N} & \% & \boldsymbol{N} & \% & \boldsymbol{N} & \% & \boldsymbol{N} & \%\end{array}$

\begin{tabular}{|c|c|c|c|c|c|c|c|c|c|c|c|c|}
\hline $\operatorname{ermA}(+)$ & 0 & 0 & 0 & 0 & 0 & 0 & 0 & 0 & 0 & 0 & 0 & 0.145744203 \\
\hline $\operatorname{ermA}(-)$ & 136 & 8 & 5.88 & 14 & 10.3 & 35 & 25.7 & 79 & 58.1 & 57 & 41.9 & \\
\hline $\operatorname{erm} B(+)$ & 98 & 7 & 7.14 & 11 & 11.2 & 29 & 29.6 & 51 & 52.0 & 47 & 48.0 & 0.122867448 \\
\hline ermB(-) & 38 & 1 & 2.63 & 3 & 7.89 & 7 & 18.4 & 27 & 71.5 & 11 & 28.9 & \\
\hline $\operatorname{erm} C(+)$ & 2 & 0 & 0 & 0 & 0 & 0 & 0 & 2 & 1.00 & 0 & 0 & 0.146326519 \\
\hline $\operatorname{erm} C(-)$ & 134 & 8 & 5.97 & 14 & 10.4 & 35 & 26.1 & 77 & 57.5 & 57 & 42.5 & \\
\hline OptrA(+) & 13 & 2 & 15.4 & 2 & 15.4 & 2 & 15.4 & 7 & 53.8 & 6 & 46.1 & 0.863018713 \\
\hline OptrA(-) & 126 & 6 & 4.76 & 12 & 9.52 & 35 & 27.8 & 73 & 57.9 & 53 & 42.1 & \\
\hline $\operatorname{Cfr}(+)$ & 0 & 0 & 0 & 0 & 0 & 0 & 0 & 0 & 0 & 0 & 0 & 0.145744203 \\
\hline $\operatorname{Cfr}(-)$ & 136 & 8 & 5.88 & 14 & 10.3 & 35 & 25.7 & 79 & 58.1 & 57 & 41.9 & \\
\hline $\operatorname{cfr} B(+)$ & 0 & 0 & 0 & 0 & 0 & 0 & 0 & 0 & 0 & 0 & 0 & 0.145744203 \\
\hline $\operatorname{cfr} B(-)$ & 136 & 8 & 5.88 & 14 & 10.3 & 35 & 25.7 & 79 & 58.1 & 57 & 41.9 & \\
\hline$m s r A(+)$ & 0 & 0 & 0 & 0 & 0 & 0 & 0 & 0 & 0 & 0 & 0 & 0.145744203 \\
\hline$m s r A(-)$ & 136 & 8 & 5.88 & 14 & 10.3 & 35 & 25.7 & 79 & 58.1 & 57 & 41.9 & \\
\hline$m s r B(+)$ & 0 & 0 & 0 & 0 & 0 & 0 & 0 & 0 & 0 & 0 & 0 & 0.145744203 \\
\hline$m s r B(-)$ & 136 & 8 & 5.88 & 14 & 10.3 & 35 & 25.7 & 79 & 58.1 & 57 & 41.9 & \\
\hline $\operatorname{mef} A E(+)$ & 30 & 6 & 20.0 & 1 & 3.33 & 3 & 10.0 & 20 & 66.7 & 10 & 33.327 & 0.094142032 \\
\hline $\operatorname{mef} A E(-)$ & 106 & 29 & 27.4 & 13 & 12.3 & 32 & 30.2 & 32 & 30.2 & 74 & 69.8 & \\
\hline $\operatorname{tet} M(+)$ & 61 & 5 & 8.20 & 3 & 4.92 & 16 & 26.2 & 37 & 60.7 & 24 & 39.3 & 0.74704373 \\
\hline $\operatorname{tet} M(-)$ & 75 & 3 & 4.00 & 11 & 14.7 & 19 & 25.3 & 42 & 56.0 & 33 & 44.0 & \\
\hline $\operatorname{tet} O(+)$ & 46 & 2 & 4.34 & 7 & 15.2 & 12 & 26.1 & 25 & 54.3 & 21 & 45.7 & 0.504670116 \\
\hline $\operatorname{tet} O(-)$ & 90 & 6 & 6.67 & 7 & 1.11 & 23 & 25.6 & 54 & 60.0 & 36 & 40.0 & \\
\hline $\operatorname{tet} K(+)$ & 27 & 1 & 3.70 & 2 & 7.40 & 10 & 37.0 & 14 & 51.9 & 13 & 48.1 & 0.696589923 \\
\hline $\operatorname{tet}(-)$ & 109 & 7 & 6.42 & 12 & 11.0 & 25 & 22.9 & 65 & 59.6 & 44 & 40.4 & \\
\hline
\end{tabular}

\section{Declarations}

\section{Data availability}


The RNA-sequencing data were deposited in the NCBI database (https://www.ncbi.) with BioSample of qRT-PCR.

\section{Funding}

This work was supported by the grants from provincial medical funds of Guangdong in China(No. 2018A0303130031).

\section{Author contributions}

Zhijian Yu participated in the design of the study and drafted the manuscript. Qiwen Deng, Zhijian Yu, and Guiqiu Li were involved in RNA-sequencing analysis, qRT-PCR assays and performed the antibiotic susceptibility tests. Ying Wei, Yan Guo, Jie Lian囚Shaoyuan WuखBing Bai and Guangjian Xu performed the PCR detections for MLST. Zhijian Yu凶Qiwen Deng and Guiqiu Li designed the study and revised the manuscript.

\section{Transparency declarations}

The authors declare that they have no conflicts of interest.

\section{References}

[1] L. Thomas, L. Cook. Two-Component Signal Transduction Systems in the Human Pathogen, Streptococcus Agalactiae. Infection and Immunity. 2020

[2] Limin Li, Xianhua Wu,Lifeng Xu. Drug resistance and clinical infection distribution of Streptococcus agalactiac. Chinese Journal of Nosocomiology. 2014, 024 (003): 549-551

[3]Man Li. Influence of several resistance genes on virulence of Strepufcoccus agalactiae and Streptococcus dysgalactiae. Inner Mongolia Agricultural University. 2015

[4] DingShu Shen,Xueyan Zhou. Advances of Streptococcus agalactiae.Chinese Journal Of Microecology. 2008, 20 (5): 518-519

[5] Yongchun, Yang, Yinglong, Liu, Yunlei, Ding, Li, Yi, Zhe, Ma. Molecular Characterization of Streptococcus Agalactiae Isolated from Bovine Mastitis in Eastern China. Plos One. 2013

[6] F.S. Nurani, S. Sukenda, S. Nuryati. Maternal Immunity of Tilapia Broodstock Vaccinated with Polyvalent Vaccine and Resistance of Their Offspring against Streptococcus Agalactiae. Aquaculture Research. 2020 (6)

[7] Z. Zhang, F. Yang, X.-p. Li, J.-y. Luo, H.-s. Li. Distribution of Serotypes, Antimicrobial Resistance and Virulence Genes among Streptococcus Agalactiae Isolated from Bovine in China. 2019

[8] A. Revathy, N. Ahmad, R.M. Yasin. Erythromycin Resistance and the Detection of the Erm(B) and Mef(a) Virulent Genes in Streptococcus Pneumonia Isolates from Malaysia. In: 8TH International Symposium on Antimicrobial Agents and Resistance, 2011

[9] G. Di Bonaventura, A. Pompilio, M. Monaco, F.P. de Araujo, L. Baldassarri, A. Pantosti, G. Gherardi. Adhesion and Biofilm Formation by Staphylococcus Aureus Clinical Isolates under Conditions Relevant to the Host: 
Relationship with Macrolide Resistance and Clonal Lineages. Journal of Medical Microbiology. 2019, 68 (2): 148160

[10] B. Bai, Z.W. Lin, Z.Y. Pu, G.J. Xu, F. Zhang, Z. Chen, X. Sun, J.X. Zheng, P.Y. Li, Q.W. Deng, Z.J. Yu. In Vitro Activity and Heteroresistance of Omadacycline against Clinical Staphylococcus Aureus Isolates from China Reveal the Impact of Omadacycline Susceptibility by Branched-Chain Amino Acid Transport System li Carrier Protein, Na/Pi Cotransporter Family Protein, and Fibronectin-Binding Protein. Frontiers in Microbiology. 2019, 10: 12

[11] A.F. M, S. George, T. Evan, M. Amy, S. Judith, D.A. F, E.P. B, M.P. C. Omadacycline for Acute Bacterial Skin and Skin Structure Infections. Clinical Infectious Diseases. 2019 (Supplement_1): Supplement_1

[12] Kenneth, LaPensee, Rohit, Mistry, Thomas, Lodise. Budget Impact of Omadacycline for the Treatment of Patients with Community-Acquired Bacterial Pneumonia in the United States from the Hospital Perspective. American Health \& Drug Benefits. 2019

[13] L. Z, P. Z, X. G, B. B, C. Z, S. X, Z. J, L. P, Q. D, D. Q. Omadacycline Efficacy against Enterococcus Faecalis Isolated in China: In Vitro Activity, Heteroresistance, and Resistance Mechanisms. Antimicrobial agents and chemotherapy. 2020, 64 (3)

[14] N.K. Kondrasheva, V.A. Rudko, D.O. Kondrashev, V.S. Shakleina, K.I. Smyshlyaeva, R. Konoplin, A. Shaidulina, A. Ivkin, I. Derkunskii, O. Dubovikov. Application of a Ternary Phase Diagram to Describe the Stability of Residual Marine Fuel. Energy \& Fuels. 2019

[15] X. Zhi-chao, Z. Bo, Z. Wan-ying, C. Xian-di, L. Duo-yun, W. Hong-yan, C. Zhong, B. Bing, P. Wei-gaung, L. Guiqiu. The Distribution of Virulence Factors in Streptococcus Agalactiae and Its Relationship to Antimicrobial Resistance and Mlst. journal of pathogen biology. 2018

\section{Figures}




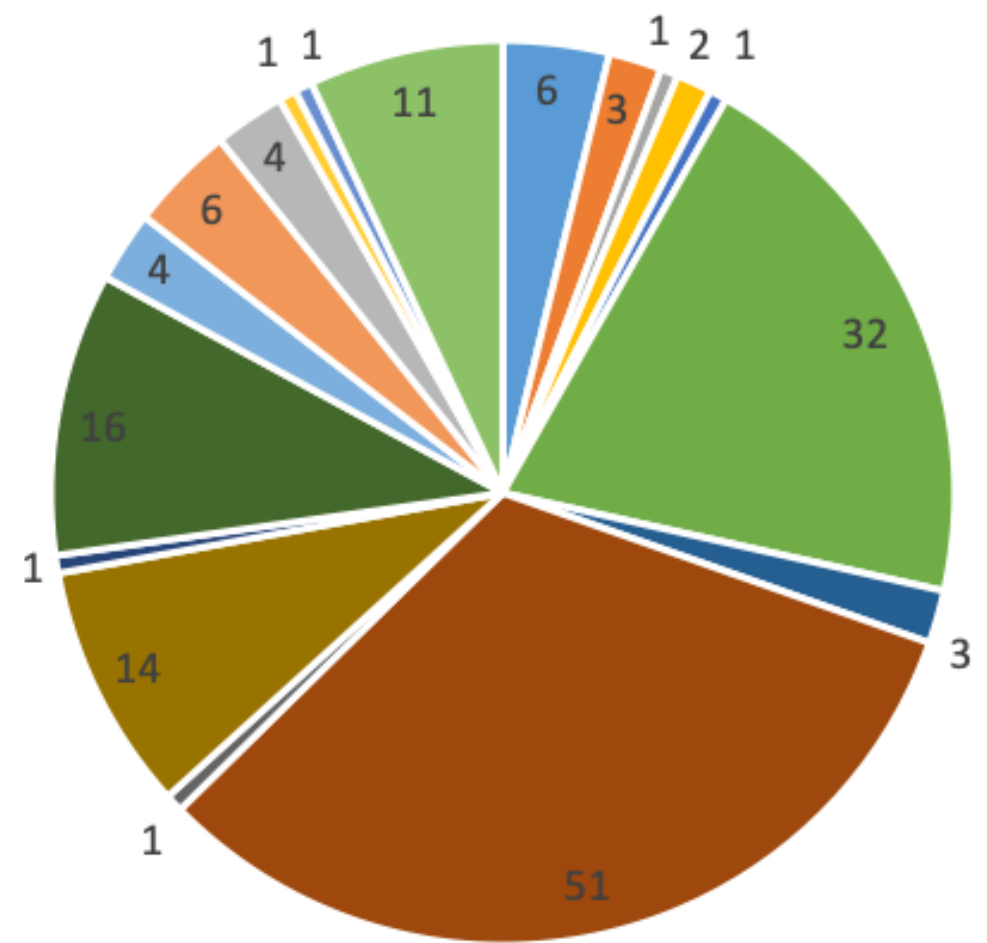

- Cervical secretions

- Wound secretions

Ear swab

n Secretions

- semen

- Venous blood

- Cerebrospinal fluid

- Pee

- Urethral discharge

- Pus

- Umbilical secretions

- Wound secretions

- Reproductive tract secretions = sputum

- Gastric juice

- Throat swab

- Eye secretions

- Amniotic fluid

Figure 1

The clinical sample type of S. agalactiae strains 